\title{
BMJ Open Predictors of postpartum glucose intolerance in women with gestational diabetes mellitus: a prospective cohort study in Ethiopia based on the updated diagnostic criteria
}

\author{
Achenef Asmamaw Muche (D) ,1,2 Oladapo O Olayemi, ${ }^{3}$ Yigzaw Kebede Gete ${ }^{2}$
}

To cite: Muche AA, Olayemi 00, Gete YK. Predictors of postpartum glucose intolerance in women with gestational diabetes mellitus: a prospective cohort study in Ethiopia based on the updated diagnostic criteria. BMJ Open 2020;10:e036882. doi:10.1136/ bmjopen-2020-036882

- Prepublication history for this paper is available online. To view these files, please visit the journal online (http://dx.doi org/10.1136/bmjopen-2020036882).

Received 08 January 2020 Revised 16 June 2020 Accepted 23 June 2020

Check for updates

(c) Author(s) (or their employer(s)) 2020. Re-use permitted under CC BY-NC. No commercial re-use. See rights and permissions. Published by BMJ.

${ }^{1}$ Pan African University Life and Earth Sciences Institute (including health and agriculture), Department of Obstetrics and Gynaecology, College of Medicine, University of Ibadan, Ibadan, Nigeria ${ }^{2}$ Department of Epidemiology and Biostatistics, University of Gondar, Gondar, Ethiopia ${ }^{3}$ Obstetrics and Gynaecology, University College Hospital Ibadan, Ibadan, Nigeria

Correspondence to Dr Achenef Asmamaw Muche; Ashua2014@gmail.com

\section{ABSTRACT}

Objectives To identify the incidence of postpartum glucose intolerance and develop a prediction model based on antenatal characteristics to predict postpartum glucose intolerance.

Design Prospective cohort study.

Setting Gondar town public health facilities in Northwest Ethiopia.

Participants Women who had gestational diabetes mellitus were advised to undergo postpartum oral glucose tolerance test at 6-12 weeks of delivery.

Main outcome Postpartum glucose intolerance.

Data analysis Predictors of postpartum glucose intolerance were identified using multivariable logistic regression analysis. The discriminative power of the predictor variables for postpartum glucose intolerance and the model accuracy were computed by area under the receiver operating characteristic curve and estimated by area under the curve (AUC) with $95 \% \mathrm{Cl}$.

Results A total of 112 (85.5\%) women with gestational diabetes mellitus returned and completed the postpartum oral glucose tolerance test. The incidence of postpartum glucose intolerance was $21.4 \%$ (95\% Cl14.3 to 28.4), inclusive of $18.7 \%$ pre-diabetes and $2.7 \%$ diabetes. Multivariable logistic regression analysis revealed that advanced maternal age, high fasting plasma glucose level at diagnosis, overweight and/or obesity, and antenatal depression were predictors of postpartum glucose intolerance. The AUC of the final reduced model to predict postpartum glucose intolerance was $0.884(95 \% \mathrm{Cl}$ 0.822 to 0.937 ). Fasting plasma glucose at diagnosis of gestational diabetes mellitus (AUC $=0.736,95 \% \mathrm{Cl} 0.616$ to 0.845 ) and overweight and/or obesity (AUC $=0.718,95 \%$ $\mathrm{Cl} 0.614$ to 0.814 ) were better predictors of postpartum glucose intolerance. Moreover, the AUC for the combined predictors of fasting plasma glucose at diagnosis and mid-upper arm circumference was $0.822(95 \% \mathrm{Cl} 0.722$ to 0.907), which was the best predictor.

Conclusions The incidence of postpartum glucose intolerance was high among women with gestational diabetes mellitus. Antenatal predictors modestly predicted postpartum glucose intolerance. The findings suggest ongoing glucose screening is indicated for all women with gestational diabetes mellitus.
Strengths and limitations of this study

- This prospective cohort study involved women with gestational diabetes mellitus diagnosed using the updated diagnostic criteria and were followed until 6-12 weeks of delivery.

- The prediction model is constructed from easily obtainable antenatal characteristics, making it applicable to low-resource settings.

- The study used a relatively small sample size.

\section{INTRODUCTION}

Gestational diabetes mellitus (GDM) is defined as 'hyperglycemia first detected during pregnancy that is clearly not preexisting or overt diabetes'. 'Although GDM normally disappears after birth, women previously diagnosed with the disease are at high risk of developing long-term metabolic disorders such as type 2 diabetes. ${ }^{2-5}$

Postprandial hyperglycaemia is common among women with GDM, and more than half develop type 2 diabetes 5 years after delivery. ${ }^{67}$ A systematic review conducted by Kim $e t a l^{8}$ disclosed that the incidence of type 2 diabetes among women with prior GDM ranged from $2.6 \%$ to $70 \%$. Similarly, the prospect of the incidence of diabetes was also high at succeeding pregnancies with GDM. ${ }^{8}$

The international guidelines recommend that women with GDM should be screened for persistent diabetes at 6-12 weeks post partum. ${ }^{9}{ }^{10}$ Indeed, the identification of women who are at the highest risk of progressing to postpartum glucose intolerance in our setting remains limited. In resource-limited settings, pregnancy often marks the first formal exposure to healthcare. The identification of predictors for potential future progression to pre-diabetes and/or diabetes in women with GDM could 
Open access

Table 1 Characteristics of patients with GDM according to postpartum glucose test results

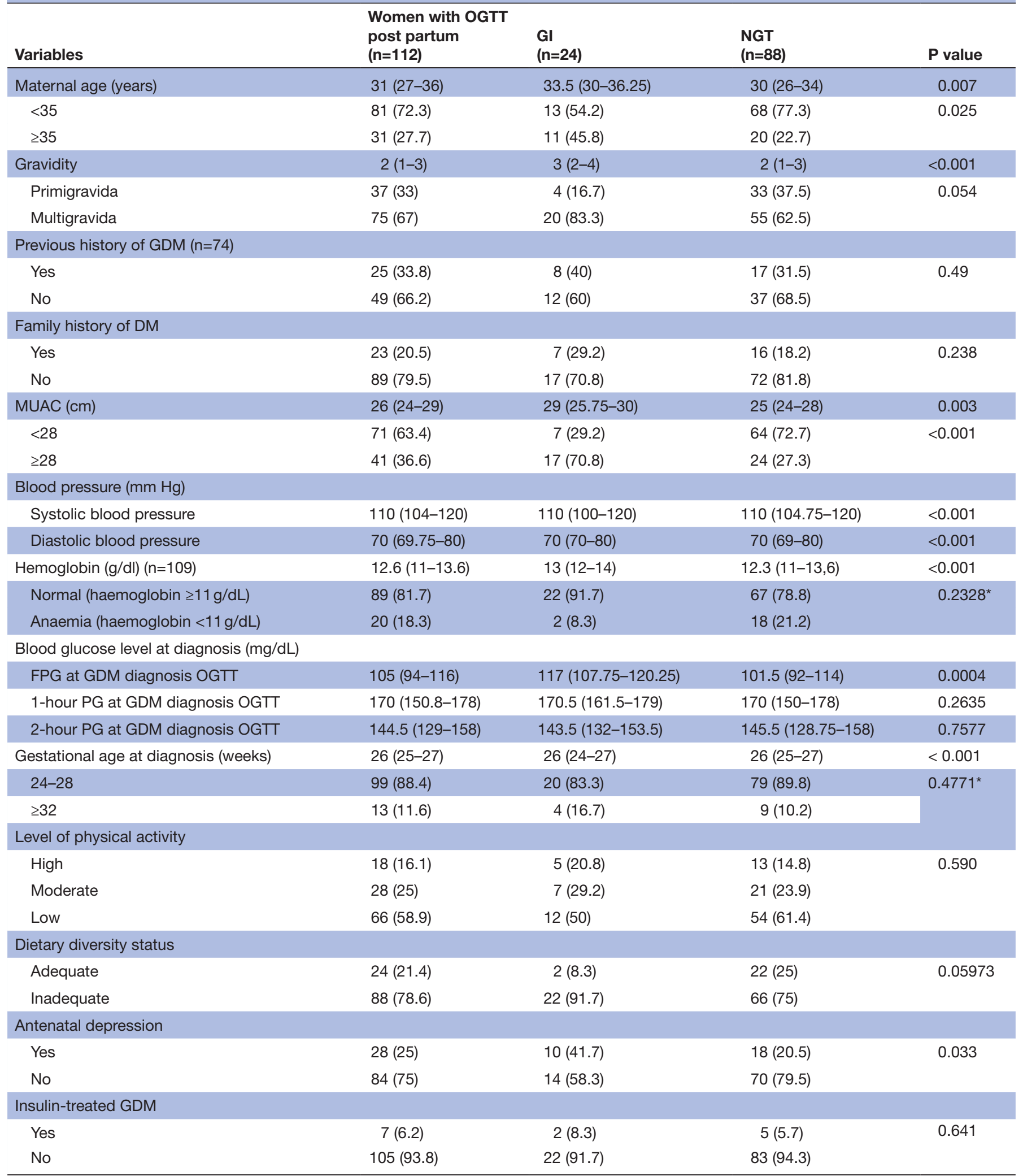

Data are presented by $\mathrm{n}(\%)$ or median (IQR).

${ }^{*} \mathrm{P}$ value of Fisher exact test

DM, diabetes mellitus; FP, Plasma glucose; FPG, fasting plasma glucose; GDM, gestational diabetes mellitus; Gl, glucose intolerance; MUAC, midupper arm circumference; NGT, normal glucose tolerance; OGTT, oral glucose tolerance test. 
Table 2 Multiple logistic regressions for predicting postpartum glucose intolerance among women with GDM

\begin{tabular}{|c|c|c|c|c|}
\hline \multirow[b]{2}{*}{ Predictor variables } & \multirow{2}{*}{$\begin{array}{l}\text { Univariable analysis } \\
\text { COR }(95 \% \mathrm{Cl})\end{array}$} & \multicolumn{2}{|c|}{ Multivariable analysis } & \multirow{2}{*}{$\begin{array}{l}\text { Simplified risk } \\
\text { score }\end{array}$} \\
\hline & & AOR $(95 \% \mathrm{Cl})$ & $P$ value & \\
\hline Maternal age ( $\geq 35$ years) & $2.88(1.111 .7 .46)$ & $4.04(1.2314 .33)$ & 0.02380 & 4 \\
\hline Gravidity (multigravida)* $^{\star}$ & $3.00(1.027$ 10.989) & $1.75(0.398 .66)$ & 0.47196 & - \\
\hline History of GDM & $1.45(0.454 .19)$ & NA & & \\
\hline Family history of DM & $1.85(0.635 .11)$ & NA & & \\
\hline $\operatorname{MUAC}(\geq 28 \mathrm{~cm})$ & $6.48(2.47418 .61)$ & $3.92(1.13,15.04)$ & 0.03617 & 4 \\
\hline \multicolumn{5}{|l|}{ Blood pressure $(\mathrm{mm} \mathrm{Hg})$} \\
\hline Systolic blood pressure & 0.998 (0.976 1.054) & NA & & \\
\hline Diastolic blood pressure & $1.015(0.9761 .053)$ & NA & & \\
\hline Anaemia (haemoglobin $<11 \mathrm{~g} / \mathrm{dL}$ ) & $0.34(0.051 .30)$ & NA & & \\
\hline \multicolumn{5}{|l|}{ Blood glucose level (mg/dL) } \\
\hline FPG at GDM diagnosis & $1.07(1.031 .13)$ & $1.08(1.041 .15)$ & 0.00171 & 1 \\
\hline 1-hour PG at GDM diagnosis & $1.014(0.991 .03)$ & NA & & \\
\hline 2-hour PG at GDM diagnosis & $0.99(0.971 .02)$ & NA & & \\
\hline Gestational age at diagnosis (weeks) & $1.03(0.891 .18)$ & NA & & \\
\hline \multicolumn{5}{|l|}{ Level of physical activity } \\
\hline High & 1 & & & \\
\hline Moderate & $0.87(0.233 .47)$ & NA & & \\
\hline Low & $0.57(0.182 .07)$ & & & \\
\hline Inadequate dietary diversity* & $3.66(0.9724 .03)$ & $3.07(0.5824,45)$ & 0.22031 & - \\
\hline Antenatal depression & $2.78(1.057 .29)$ & $5.90(1.66$ 23.47) & 0.00770 & 5 \\
\hline Insulin-treated GDM & $1.51(0.217 .54)$ & NA & & \\
\hline
\end{tabular}

*Gravidity and dietary diversity status were variables that were also retained in the reduced model using likelihood ratio test. Both backward and forward selection showed the same results. ORs after internal validation with bootstrapping are shown.

AOR, adjusted OR; COR, crude OR; DM, diabetes mellitus; FPG, fasting plasma glucose; GDM, gestational diabetes mellitus; MUAC, midupper arm circumference; NA, not included in the multivariate analysis .

improve accurate risk stratification of patients during pregnancy. This provides an opportunity for appropriate, cost-effective and priority intervention programmes for high-risk groups. If the persistence risk can be estimated accurately, treatment may be tailored to individual patient needs. Low persistence risk warrants adoption of a watchful waiting policy, while a high persistence risk may call for immediate and possibly more appropriate management (eg, lifestyle modification and behavioural change in combination with drug treatment).

Although there are few available studies that determine the risk factors for postpartum glucose intolerance, they presently do not allow prediction of the absolute risk in individual patients in daily practice. ${ }^{11-14}$ It is anticipated that our setting could use such models to predict the risk of postpartum glucose intolerance in women with GDM and to refer patients early. This predictive model could help prospectively evaluate and determine the presence of persistent diabetes, and guide caregivers in promptly providing the best treatment choice for individual patients and be more cost-effective by identifying high-risk patients who will benefit most from certain interventions. We sought to (1) identify the incidence of postpartum glucose intolerance and (2) develop a prediction model to enable objective estimations of outcome probabilities (risks) according to different combinations of predictor values for women with GDM in the Ethiopian context using the updated international diagnostic criteria. We hypothesised that using antenatal clinical characteristics would improve the identification of women with GDM at high risk for postpartum glucose intolerance.

\section{METHODS AND MATERIALS}

This prospective cohort study was part of a larger project, where similar methodology was used in previous published article elsewhere. ${ }^{15}$

\section{Study design and population}

This study was conducted in five selected public health facilities of Gondar town, namely University of Gondar Comprehensive Specialized Hospital and Health Centers (Gondar, Woleka, Azezo and Maraki), from 30 March 2018 to 26 March 2019. Pregnant women were enrolled if they were 18 years or older, had singleton pregnancy and at $20-23^{+6}$ weeks' gestation during commencement 
Table 3 Sensitivity and specificity of the FPG test for postpartum glucose intolerance

\begin{tabular}{|c|c|c|c|c|c|c|}
\hline $\begin{array}{l}\text { Threshold } \\
\text { FPG } \\
\text { (mg/dL) }\end{array}$ & $\begin{array}{l}\text { Sensitivity } \\
\%(95 \% \mathrm{CI})\end{array}$ & $\begin{array}{l}\text { Specificity } \\
\%(95 \% \text { Cl) }\end{array}$ & $\begin{array}{l}\text { LR+ } \\
(95 \% \mathrm{Cl})\end{array}$ & $\begin{array}{l}\text { LR- } \\
(95 \% \mathrm{CI})\end{array}$ & $\begin{array}{l}\text { Positive } \\
\text { post-test } \\
\text { probability } \\
\%(95 \% \mathrm{Cl})\end{array}$ & $\begin{array}{l}\text { Negative } \\
\text { post-test } \\
\text { probability } \\
\%(95 \% \mathrm{Cl})\end{array}$ \\
\hline$\geq 116$ & 54 (33 to 74$)$ & 78 (68 to 86$)$ & 2.51 (1.46 to 4.31$)$ & 0.58 (0.37 to 0.92) & 41 (24 to 59$)$ & 86 (77 to 93 ) \\
\hline$\geq 105$ & 79 (58 to 93) & 56 (45 to 66$)$ & 1.79 (1.31 to 2.44$)$ & $0.37(0.17$ to 0.83$)$ & 33 (21 to 46$)$ & 91 (80 to 97$)$ \\
\hline$\geq 94$ & 88 (68 to 97$)$ & 27 (18 to 38$)$ & $1.20(0.99$ to 1.47$)$ & $0.46(0.15$ to 1.39$)$ & 25 (16 to 35$)$ & 89 (71 to 98$)$ \\
\hline
\end{tabular}

FPG, fasting plasma glucose; LR-, negative likelihood ratio; LR+, positive likelihood ratio.

time, and were followed until 6-12 weeks after delivery. Pregnant women who had pre-existing or overt diabetes, chronic diseases, or on medications that might affect glucose metabolism (steroids, $\beta$-adrenergic agonists, antipsychotic drugs) at commencement were excluded. All pregnant women were screened for overt diabetes at their first antenatal care visit. If the test at the first visit was normal, a 2-hour $75 \mathrm{~g}$ oral glucose tolerance test (OGTT) was performed for all pregnant women at 24-28 weeks' gestation to screen for GDM. High-risk women were advised to repeat the test at 32-36 weeks even if their OGTT results were normal at 24-28 weeks' gestation. As described earlier among 1027 pregnant women, $131(12.8 \%)$ were diagnosed with GDM..$^{15}$ All women with GDM were invited to participate in the study and their glucose status was evaluated at 6-12 weeks post partum.

\section{Data collection}

All women who had GDM were encouraged to return for postpartum glucose tolerance test. The baseline data of each participant were linked to these study data. Data included demographic profile, obstetric history, anthropometric measurements, type of treatment of GDM (diet or insulin), behavioural factors (alcohol use and coffee intake), lifestyle parameters (dietary diversity and physical activity), antenatal depression status and blood glucose value (fasting plasma glucose (FPG) and OGTT). All participants also had FPG and 2-hour OGTT blood tests performed at 6-12 weeks after delivery.

\section{Laboratory assessment}

The universal screening for GDM using a 2-hour $75 \mathrm{~g}$ OGTT was performed for all pregnant women by capillary glucose testing, using a standard plasma-calibrated glucometer (HemoCue Glucose B-201+, Ängelholm, Sweden). This corresponded to the latest consensus recommendations of the International Federation of Gynecology and Obstetrics initiative for GDM diagnosis in settings where close-by laboratories or facilities for proper storage and transport of blood samples to distant laboratories are not available. ${ }^{16}$ After capillary blood samples were taken, the whole blood capillary values were converted to plasma venous values by multiplying with a constant factor of $1.11 .{ }^{17}$ The updated diagnostic criteria for GDM diagnosis were made according to the 2017 American Diabetes Association, ${ }^{18} 2013$ WHO, ${ }^{1}$ or modified International
Association of Diabetes and Pregnancy Study Groups. ${ }^{19}$ The diagnosis of GDM is made when one or more of the values of plasma glucose level were met (fasting: $\geq 92 \mathrm{mg}$ / dL; 1 hour: $\geq 180 \mathrm{mg} / \mathrm{dL} ; 2$ hours: $\geq 153 \mathrm{mg} / \mathrm{dL}$ ). Similarly, postpartum glucose tolerance status was evaluated using standard FPG and $75 \mathrm{~g}$ 2-hour OGTT, with a similar test procedure but a higher cut-off point for classification of postpartum glucose intolerance. ${ }^{118}$

\section{Outcome measures}

The primary outcome was diagnosis of postpartum pre-diabetes (impaired fasting glucose (IFG): FPG $100-125 \mathrm{mg} / \mathrm{dL}$; impaired glucose tolerance (IGT): 2-hour plasma glucose in 75g OGTT $140-199 \mathrm{mg} /$ $\mathrm{dL}$ ) or diabetes (FPG $\geq 126 \mathrm{mg} / \mathrm{dL}$, or 2-hour plasma glucose $\geq 200 \mathrm{mg} / \mathrm{dL}$ in OGTT or random plasma glucose $\geq 200 \mathrm{mg} / \mathrm{dL}) .{ }^{18}$ Subjects were divided into two groups: the glucose intolerance group, which consisted of IGT and IFG patients, and the normal group.

\section{Data processing and statistical analysis}

All data were entered into Epi Info V.7 software and exported to R V.3.6.0 statistical programming language for analysis. Descriptive statistics (mean, median, SD, IQR, percentages and rates) were computed. Differences in the distribution of categorical variables were analysed with $\chi^{2}$ test. The Shapiro-Wilk test was used to verify if continuous variables were normally distributed. Normally distributed and non-normally distributed variables were evaluated with t-test and Mann-Whitney test, respectively. Glycaemia on diagnostic OGTT was correlated to postpartum OGTT using the Spearman correlation test. We performed a univariable analysis using logistic regression to obtain insight into the association of each potential determinant with postpartum glucose intolerance and to select potential predictors for the multivariable analysis. We fitted all variables with $p \leq 0.2$ in the univariable analysis to the multivariable model to be more liberal. Then we used a stepwise backward elimination technique with $\mathrm{p}<0.10$ for the likelihood ratio test to fit the reduced model of easily obtainable characteristics. In this study, the significant factors have been defined as variables with $\mathrm{p}<0.05$ in the multivariable logistic regression analysis.

For the discriminative power of predictor variables of postpartum glucose intolerance and to check model accuracy, we computed the area under the receiver operating 
A

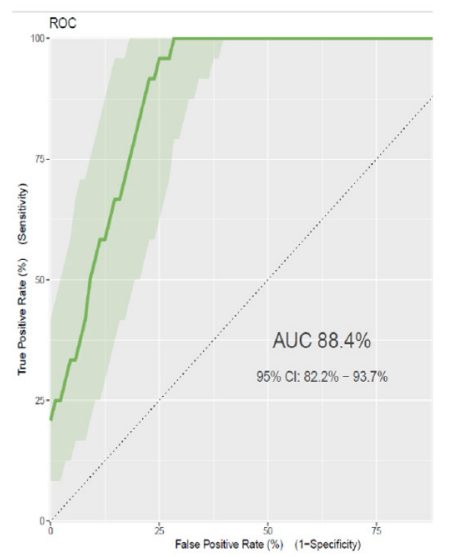

B

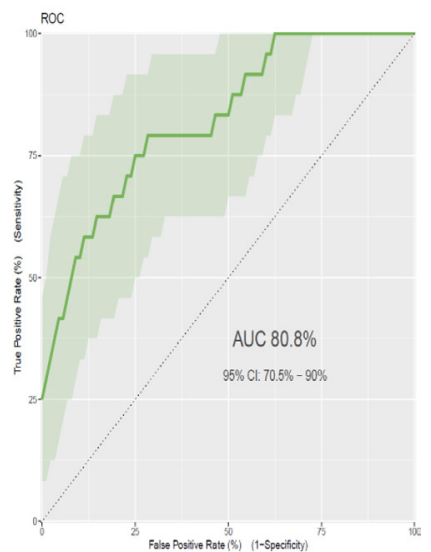

Figure 1 ROC curve for prediction of postpartum glucose intolerance using different models. (A) Linear predictor model for estimated risk of postpartum glucose intolerance $=1 /$ $(1+$ exp $-(-11.87007)+1.48 \times$ age $(\geq 35$ years $)+1.716 \times$ overweight and/or obesity (MUAC $\geq 28 \mathrm{~cm})+0.081 \times \mathrm{FPG}$ at diagnosis $+1.637 \times$ antenatal depression (yes)). (B) Simplified risk score predictor model for estimated risk of postpartum glucose intolerance $=($ age $\geq 35$ years $\times 4)+($ overweight and/ or obesity (MUAC $\geq 28 \mathrm{~cm}) \times 4)+($ FPG at diagnosis $\times 1)$ $+($ antenatal depression $\times 5)$. AUC, area under the curve; FPG, fasting plasma glucose; MUAC, mid-upper arm circumference; ROC, receiver operating characteristic.

characteristic (ROC) curve (discrimination) and calibration plot (calibration) using 'classifierplots' and 'givitiR' packages of $\mathrm{R}$, respectively, ${ }^{20}$ and estimated as the area under the curve (AUC) with 95\% CI. The AUC ranged from 0.5 (no predictive ability) to 1 (perfect discrimination). To construct an easily applicable postpartum glucose intolerance prediction score, we transformed each coefficient from the model to a round number by dividing with the lowest coefficient. The number of points was subsequently rounded to the nearest integer. We determined the total score for everyone by assigning the points to each variable present and adding them up. In addition, sensitivity, specificity, likelihood ratios and post-test probability of FPG at diagnosis, with $95 \%$ CI, were calculated using the optimal cut-offs of levels.

\section{Patient and public involvement}

Patients and the public were not invited to comment on study design or conduct of the study. However, they will be informed of the study results through publications.

\section{RESULTS}

\section{Characteristics of the study group}

Of all 131 women with GDM, $112(85.5 \%)$ returned and completed the postpartum OGTT at 6-12 weeks after delivery. The incidence of early postpartum glucose intolerance was $21.4 \%$ (95\% CI 14.3 to 28.4 ), inclusive of $18.7 \%$ (95\% CI 11.5 to 25.3 ) pre-diabetes and $2.7 \%$ (95\% CI 0.9 to 6.4 ) diabetes.

The median age of women was 31 (IQR: 27-36) years, $20.5 \%$ had family history of diabetes mellitus, 33.8\%

had history of GDM, $18.3 \%$ were anaemic, and $36.6 \%$ were overweight and/or obese at the first prenatal visit. A higher proportion of overweight and/or obesity $(p<0.001)$, maternal age of $\geq 35$ years $(p=0.025)$ and antenatal depression $(p=0.033)$ were seen among women with postpartum glucose intolerance than those with normal glucose profile (table 1).

There was a positive correlation between FPG during pregnancy and postpartum FPG $(\mathrm{r}=0.424, \mathrm{p}<0.001)$. There was also a positive correlation between 2-hour plasma glucose level during pregnancy and 2-hour postpartum plasma glucose level $(\mathrm{r}=0.213, \mathrm{p}=0.024)$.

\section{A prediction model for postpartum glucose intolerance}

Different demographic, obstetric, and clinical characteristics of mothers were collected during prenatal visits and were considered to predict postpartum glucose intolerance. On univariable analysis, maternal age, gravidity, maternal obesity and/or overweight, FPG at GDM diagnosis, and antenatal depression were found to have a significant association. However, in the final multivariable regression analysis and the reduced model, four predictors of progression, namely age of the mother ( $\geq 35$ years) during pregnancy $(\mathrm{AOR}=4.04,95 \%$ CI 1.23 to 14.33), maternal obesity and/or overweight (AOR $=3.92,95 \%$ CI 1.13 to 15.04$)$, FPG at GDM diagnosis $(\mathrm{AOR}=1.08,(95 \%$ CI 1.04 to 1.15$)$, and antenatal depression $(\mathrm{AOR}=5.90$, (95\% CI 1.66 to 23.47), remained significant. Using the results, a prediction model was developed and an equation for the prediction model was obtained (table 2).

The area under the ROC curve of the final reduced model was 0.884 (95\% CI: 0.822 to 0.937$)$. The calibration test had a $\mathrm{p}$ value of 0.759 , indicating the model does not misrepresent the data (figure 1A). Rounding of all regression coefficients in the reduced model to one point resulted in a simplified prediction score, which is presented in table 2. The AUC of the simplified risk score prediction model was 0.808 (95\% CI 0.705 to 0.90 ). The calibration test had a $\mathrm{p}$ value of 0.044 , indicating the model less represented the data (figure 1B). Since the simplified score had a lower prediction accuracy than the model that used the results of the original $\beta$ coefficients, we prefer to use the original $\beta$ coefficients.

In addition, to verify whether any antepartum trait was used as a specific predictor of postpartum glucose intolerance we performed an ROC analysis. The analysis indicated that FPG at GDM diagnosis $(\mathrm{AUC}=0.736,95 \%$ CI 0.616 to $0.845, \mathrm{p}<0.001$ ), overweight and/or obesity (AUC $=0.718,95 \%$ CI:0.614 to $0.814, p=0.0284$ ), maternal age ( $\geq 35$ years) (AUC $=0.616,95 \%$ CI 0.506 to 0.722 , $\mathrm{p}<0.001$ ), and antenatal depression $(\mathrm{AUC}=0.606,95 \%$ CI 0.506 to $0.718, p=0.0375$ ) emerged as better predictors of postpartum glucose intolerance (figure 2). Moreover, the AUC for the combined predictors of FPG at diagnosis and mid-upper arm circumference (MUAC) was $0.822(95 \%$ CI 0.722 to 0.907 ), for FPG at diagnosis and antenatal depression was 0.793 (95\% CI 0.698 to 0.876 ), and for MUAC and antenatal depression was 0.759 (95\% CI 0.646 


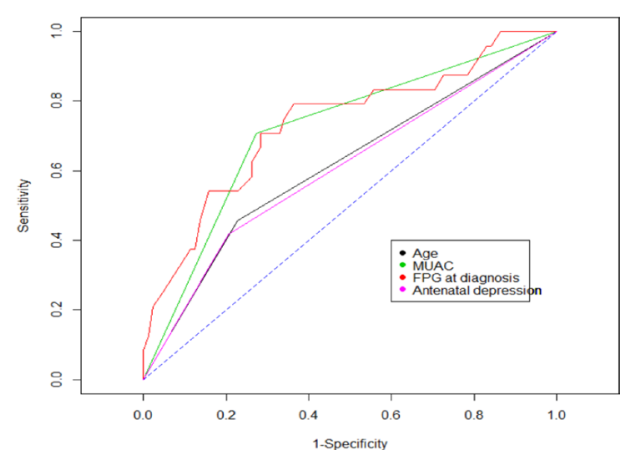

Figure 2 Receiver operating characteristic curve of antepartum parameters for prediction of postpartum glucose intolerance. FPG, fasting plasma glucose; MUAC, mid-upper arm circumference.

to 0.856 ) (figure 3 ). The evaluation of sensitivity across different FPG level thresholds showed that FPG $\geq 105 \mathrm{mg} /$ dL during pregnancy had an optimal sensitivity of $79 \%$ (95\% CI $58 \%$ to $93 \%)$, with a specificity of $56 \%$ (95\% CI $45 \%$ to $66 \%$ ), in predicting postpartum glucose intolerance (table 3 ).

\section{DISCUSSION}

This prospective study aimed to identify glucose status at an early postpartum stage after diagnosis of GDM and the predictors of postpartum glucose intolerance. Based on recent guidelines, $21.4 \%$ of women with GDM had postpartum glucose intolerance at 6-12 weeks after delivery. The major predictors of developing glucose intolerance were advanced maternal age, overweight and/or obesity, high FPG at GDM diagnosis, and antenatal depression. Women recently diagnosed with GDM were at higher risk of developing postpartum hyperglycaemia. Accordingly, this study suggested close follow-up of women who had GDM and identification of postpartum glucose intolerance predictors as a crucial way to manage early the future risk of type 2 diabetes or delay its onset.

Our study showed that more than one-fifth of women with GDM developed early postpartum glucose intolerance. This rate was consistent with studies from Australia, ${ }^{21}$ Belgium, ${ }^{22}$ Japan $^{12}$ and Brazil. ${ }^{11}$ However, it was lower than two existing evidence in Saudi Arabia, where the prevalence of glucose intolerance was $38.6 \%{ }^{14}$ and $56 \%,{ }^{23}$ and in Belgium which was $43.7 \% .{ }^{13}$ This difference might be due to the use of different screening and diagnostic methods. We used the universal one-step approach with a $75 \mathrm{~g}$ OGTT and the updated diagnostic criteria, whereas the other studies used the universal two-step screening strategy for GDM. ${ }^{13}$ The two-step screening strategy with a glucose challenge test, therefore, has the potential to limit the number of OGTTs to screen for GDM and identify a high-risk group for postpartum glucose intolerance. The strong association between GDM and postpartum glucose intolerance indicates that the course of the disease developed at an early stage. ${ }^{24}{ }^{25}$ Pregnancy itself caused insulin resistance, and hyperglycaemia can occur as a result of

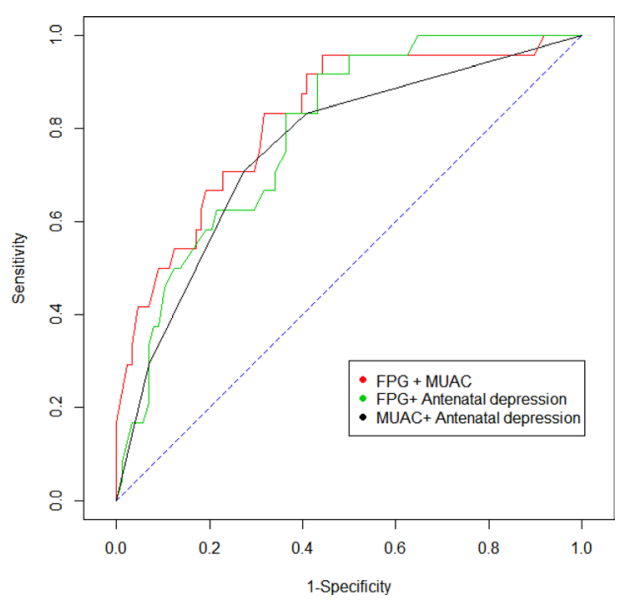

Figure 3 Receiver operating characteristic curve for combined predictors of FPG at diagnosis, MUAC and antenatal depression for prediction of glucose intolerance. FPG, fasting plasma glucose; MUAC, mid-upper arm circumference.

this metabolic change. ${ }^{26}{ }^{27}$ In addition, the early onset of GDM indicated the presence of pregestational insulin resistance and/or pancreatic B-cell dysfunction, which leads to higher risk of postpartum glucose abnormality. ${ }^{21}$ This finding highlights the importance of improving the uptake of blood glucose checks and lifestyle modifications before the onset of type 2 diabetes. Regardless of which screening approach is used, research on the efficacy or effectiveness of lifestyle interventions for preventing or delaying progression to postpartum glucose intolerance after GDM in our setting would provide much needed data.

This study has shown antenatal characteristics modestly predicted the development of postpartum glucose intolerance. FPG at GDM diagnosis, MUAC and antenatal depression or combined were good predictors of postpartum glucose intolerance. The model for combined antenatal predictors resulted in an AUC of 0.88, which means the model has the best predictive ability. This prognostic prediction model provides a powerful tool for identification of patients with GDM at higher risk of occurrence of progression to postpartum glucose intolerance.

Similar to the findings of previous studies in Italy, ${ }^{28} \mathrm{UK}^{29}$ and Sweden, ${ }^{30}$ the current study has shown that FPG level in antepartum OGTT was the strongest predictor of early postpartum glucose intolerance. Evidence also revealed that elevated fasting glucose level during pregnancy has been a consistent predictor of development of type 2 diabetes in women with GDM. ${ }^{8} 128$ This suggested that $\beta$-cell dysfunction in the presence of insulin resistance is a common feature of GDM. Later, the same $\beta$-cell failure might complicate the tendency of persistent diabetes. ${ }^{26}$ Thus, the diagnosis of GDM represents a window of opportunity to implement interventions for women with high blood glucose level during antenatal visits to prevent subsequent diabetes mellitus. Moreover, this estimate has clinical utility in targeting women for early screening and prevention of subsequent diabetes. 
We found that advanced maternal age during pregnancy is a predictor of risk of abnormal glucose tolerance at 6-12 weeks post partum. Similar evidence was found in Italy ${ }^{28}$ and South Africa, ${ }^{31}$ which described advanced maternal age as a predictor of postpartum glucose intolerance. On the contrary, a study conducted in Belgium showed maternal age was not a predictor of early postpartum glucose intolerance. ${ }^{22}$ The presence of higher risk of insulin resistance and inadequate pancreatic $\beta$-cell response occurred due to advanced maternal age, which subsequently lead to diabetes progression. ${ }^{32}$ This finding suggested that due attention should be given to women with GDM at advanced maternal age. Positive lifestyle change during pregnancy could reduce the risk of GDM progressing to type 2 diabetes. ${ }^{33}$ As a low-cost intervention to prevent subsequent diabetes for women at advanced age, integrating behavioural counselling on nutrition and exercise into antenatal care services is recommended.

In our study, overweight and/or obesity was a strong predictor of early postpartum glucose intolerance. Similar studies have demonstrated that prepregnancy body mass index was predictive of subsequent diabetes. ${ }^{1434-36}$ Due to the current and ongoing high burden of overweight or obesity among African women, increased prevalence of diabetes is expected. Therefore, it is imperative to identify populations at elevated risk and introduce risklowering interventions such as reducing obesity and avoiding sedentary life. ${ }^{37} 38$

Another strong predictor of postpartum glucose intolerance was the presence of antenatal depression. Although studies are limited on the predictive effect of antenatal depression on postpartum glucose intolerance, existing evidence shows there is association between antenatal depression and GDM. ${ }^{15} 3940$ Previous studies also revealed depression increased the risk of type 2 diabetes. ${ }^{41}{ }^{42}$ The existence of comorbid problems of antenatal depression can lead women to poor lifestyle decisions, such as unhealthy eating, poor exercise, weight gain and poor glycaemic control, priming the progression to postpartum diabetes. ${ }^{43}$ Unfortunately, the guidelines for the treatment and management of GDM do not provide adequate evidence regarding the care of patients with comorbid situations of antenatal depression in lowresource settings.

The strength of this study was being a prospective cohort study involving patients with GDM identified using the updated diagnostic criteria with uniform protocols for all women and followed until 6-12 weeks of delivery. In addition, our prediction model is constructed from easily obtainable antenatal characteristics, making it applicable to low-resource settings. Although WHO recommends that in settings where laboratories or proper storage and transport of blood samples are not guaranteed, the use of point-of-care tests may influence the results. ${ }^{16}$ However, we used plasma-calibrated hand-held glucometer due to its convenience and acceptable reliability. Moreover, the study used a relatively small sample size, which could be a limitation.

\section{CONCLUSIONS}

Based on the updated diagnostic criteria, a high incidence rate of early postpartum glucose intolerance has been identified among women who had GDM. Antenatal characteristics (advanced maternal age, high FPG at GDM diagnosis, overweight and/or obesity, and antenatal depression) were strong predictors of postpartum glucose intolerance. This prognostic risk prediction model showed the utility of antenatal predictors in modestly predicting postpartum glucose intolerance in women with GDM. In addition, a risk score calculation based on a combination of antenatal predictors was effective but had lower accuracy than the model-based approach by original $\beta$ coefficients. Thus, our findings highlighted the need for increased awareness among women and their primary care providers regarding the importance of longterm glucose screening after pregnancies complicated by GDM.

Acknowledgements We would like to forward our sincere gratitude to the African Union Commission (AU) for funding this study, the Pan African University Life and Earth Sciences Institute (PAULESI), and the University of Ibadan (UI) for hosting the programme and the University of Gondar. We would also like to acknowledge Setema Limited for their material support (Hemocue Glucose 201 with micro cuvette). We are grateful to the University of Antwerp, Belgium and Vliruos scholarship for the manuscript preparation as part of the EBQ training programme. We appreciate Mr Hamid Yimam Hassen (PhD fellow at the University of Antwerp) for his professional assistance. Finally, we would like to extend our thanks to all data collectors, experts in the selected health facilities and the study participants.

Contributors AAM conceived and designed the study, analysed the data and prepared the manuscript. 000 and YKG assisted in the development of the research idea, analysis, interpretation and preparation of the manuscript. All authors read and approved the final manuscript

Funding This study was supported by the Pan African University (PAU), a continental initiative of the African Union Commission (AU), Addis Ababa, Ethiopia, as part of a PhD Fellowship Program in Reproductive Health Sciences. AAM received funding from PAU. The funder had no role in the design, data collection, analysis, interpretation of data and preparation of the manuscript.

Competing interests None declared.

Patient and public involvement Patients and/or the public were not involved in the design, or conduct, or reporting, or dissemination plans of this research.

\section{Patient consent for publication Not required.}

Ethics approval The study was conducted after ethical approval was obtained from the Institute for Advanced Medical Research and Training (IAMRAT), College of Medicine, University of Ibadan, Ibadan, Nigeria, with I/UCH EC Registration Number NHREC/05/01/2008a and UI/UCH Ethics Committee Number UI/EC/17/0435, and the Institutional Review Board (IRB) of the University of Gondar (ref no: 0/N/P/ RCS/05/811/2018). Permission from the Amhara Public Health Institute and the health authorities of the study sites was also received prior to the start of the study All participants signed (written or thumb-printed) informed consent form after they have received a face-to-face explanation about the objectives of the study. The collected information during the course of the research was treated with utmost confidentiality.

Provenance and peer review Not commissioned; externally peer reviewed.

Data availability statement Data are available upon reasonable request. The data sets used and/or analysed during the current study are available from the correspondence author on reasonable request.

Open access This is an open access article distributed in accordance with the Creative Commons Attribution Non Commercial (CC BY-NC 4.0) license, which permits others to distribute, remix, adapt, build upon this work non-commercially, and license their derivative works on different terms, provided the original work is properly cited, appropriate credit is given, any changes made indicated, and the use is non-commercial. See: http://creativecommons.org/licenses/by-nc/4.0/. 
ORCID iD

Achenef Asmamaw Muche http://orcid.org/0000-0001-7971-3537

\section{REFERENCES}

1 World Health Organization (WHO). Diagnostic criteria and classification of hyperglycaemia first detected in pregnancy, 2013. Available: http://apps.who.int/iris/bitstream/10665/8597/1/WHO_ NMH MND 13.2 eng.pdf

2 Mclntyre HD, Thomae MK, Wong SF, et al. Pregnancy in type 2 diabetes mellitus--problems \& promises. Curr Diabetes Rev 2009;5:190-200.

3 Holman N, Young B, Gadsby R. Current prevalence of type 1 and type 2 diabetes in adults and children in the UK. Diabet Med 2015;32:1119-20.

4 World Health Organization (WHO). Global report on diabetes: World Health organization; 2016

5 Bellamy L, Casas J-P, Hingorani AD, et al. Type 2 diabetes mellitus after gestational diabetes: a systematic review and meta-analysis. Lancet 2009;373:1773-9.

6 Buchanan TA, Metzger BE, Freinkel N, et al. Insulin sensitivity and $\mathrm{B}$-cell responsiveness to glucose during late pregnancy in lean and moderately obese women with normal glucose tolerance or mild gestational diabetes. Am J Obstet Gynecol 1990;162:1008-14.

7 Gerstein HC, Santaguida P, Raina P, et al. Annual incidence and relative risk of diabetes in people with various categories of dysglycemia: a systematic overview and meta-analysis of prospective studies. Diabetes Res Clin Pract 2007;78:305-12.

8 Kim C, Newton KM, Knopp RH. Gestational diabetes and the incidence of type 2 diabetes: a systematic review. Diabetes Care 2002;25:1862-8.

9 Metzger BE, Buchanan TA, Coustan DR, et al. Summary and recommendations of the fifth International workshop-conference on gestational diabetes mellitus. Diabetes Care 2007;30:S251-60.

10 American Diabetes Association. Standards of medical care in diabetes--2013. Diabetes Care 2013;36 Suppl 1:S11-66.

11 Weinert LS, Mastella LS, Oppermann MLR, et al. Postpartum glucose tolerance status 6 to 12 weeks after gestational diabetes mellitus: a Brazilian cohort. Arq Bras Endocrinol Metab 2014;58:197-204.

12 Kojima N, Tanimura K, Deguchi M, et al. Risk factors for postpartum glucose intolerance in women with gestational diabetes mellitus. Gynecological Endocrinology 2016;32:803-6.

13 Benhalima K, Jegers K, Devlieger R, et al. Glucose intolerance after a recent history of gestational diabetes based on the 2013 WHO criteria. PLoS One 2016;11:e0157272.

14 Wahabi H, Fayed A, Tunkar SMS, et al. Incidence and contributing factors of glucose intolerance in Saudi postpartum women: Subgroup analysis from RAHMA study. PLoS One 2019;14:e0210024.

15 Muche AA, Olayemi OO, Gete YK. Prevalence of gestational diabetes mellitus and associated factors among women attending antenatal care at Gondar town public health facilities, Northwest Ethiopia. BMC Pregnancy Childbirth 2019;19:334.

16 Hod M, Kapur A, Sacks DA, et al. The International Federation of Gynecology and Obstetrics (FIGO) Initiative on gestational diabetes mellitus: A pragmatic guide for diagnosis, management, and care. Int $J$ Gynaecol Obstet 2015;131:S173-211.

17 D'Orazio Pet al. Approved IFCC recommendation on reporting results for blood glucose (abbreviated). Clin Chem 2005;51:1573-6.

18 American Diabetes Association (ADA). Classification and diagnosis of diabetes. Sec. 2. in standards ofMedical care in Diabetesd 2017. Diabetes Care 2017;40:S11-24.

19 International Association of Diabetes and Pregnancy Study Groups Consensus Panel, Metzger BE, Gabbe SG, et al. International association of diabetes and pregnancy study groups recommendations on the diagnosis and classification of hyperglycemia in pregnancy. Diabetes Care 2010;33:676-82.

20 Finazzi S, Poole D, Luciani D, et al. Calibration belt for quality-ofcare assessment based on dichotomous outcomes. PLoS One 2011;6:e16110.
21 Ingram ER, Robertson IK, Ogden KJ, et al. Utility of antenatal clinical factors for prediction of postpartum outcomes in women with gestational diabetes mellitus (GDM). Aust N Z J Obstet Gynaecol 2017;57:272-9.

22 Benhalima K, Van Crombrugge P, Moyson C, et al. Prediction of glucose intolerance in early postpartum in women with gestational diabetes mellitus based on the 2013 WHO criteria. J Clin Med 2019;8:383.

23 Wahabi $\mathrm{H}$. Prevalence and risk factors for glucose intolerance among Saudi women with gestational diabetes. J Diabetes Res 2018;2018:1-5

24 Ben-Haroush A, Yogev Y, Hod M. Epidemiology of gestational diabetes mellitus and its association with type 2 diabetes. Diabetic Medicine 2004;21:103-13.

25 Cho YM, Kim TH, Lim S, et al. Type 2 diabetes-associated genetic variants discovered in the recent genome-wide association studies are related to gestational diabetes mellitus in the Korean population. Diabetologia 2009;52:253-61.

26 Buchanan TA. Pancreatic B-cell defects in gestational diabetes: implications for the pathogenesis and prevention of type 2 diabetes. $J$ Clin Endocrinol Metab 2001;86:989-93.

27 Buchanan TA, Xiang AH. Gestational diabetes mellitus. J Clin Invest 2005;115:485-91.

28 Capula C, Chiefari E, Vero A, et al. Prevalence and predictors of postpartum glucose intolerance in Italian women with gestational diabetes mellitus. Diabetes Res Clin Pract 2014;105:223-30.

29 Sivaraman SC, Vinnamala S, Jenkins D. Gestational diabetes and future risk of diabetes. J Clin Med Res 2013;5:92-6.

30 Ekelund M, Shaat N, Almgren P, et al. Prediction of postpartum diabetes in women with gestational diabetes mellitus. Diabetologia 2010;53:452-7.

31 Feig DS, Zinman B, Wang X, et al. Risk of development of diabetes mellitus after diagnosis of gestational diabetes. Can Med Assoc J 2008;179:229-34.

32 Jolly M, Sebire N, Harris J, et al. The risks associated with pregnancy in women aged 35 years or older. Hum Reprod 2000;15:2433-7.

33 Chivese T, Norris SA, Levitt NS. Progression to type 2 diabetes mellitus and associated risk factors after hyperglycemia first detected in pregnancy: a cross-sectional study in Cape Town, South Africa. PLoS Med 2019;16:e1002865-e.

34 Cheung NW, Helmink D. Gestational diabetes: the significance of persistent fasting hyperglycemia for the subsequent development of diabetes mellitus. J Diabetes Complications 2006;20:21-5.

35 Lobner K, Knopff A, Baumgarten A, et al. Predictors of postpartum diabetes in women with gestational diabetes mellitus. Diabetes 2006;55:792-7.

36 Åberg AB, Jönsson E, Eskilsson I, et al. Predictive factors of developing diabetes mellitus in women with gestational diabetes. Acta Obstet Gynecol Scand 2002;81:11-16.

37 Kengne AP, Bentham J, Zhou B, et al. Trends in obesity and diabetes across Africa from 1980 to 2014: an analysis of pooled populationbased studies. Int J Epidemiol 2017;46:1421-32.

38 Puoane T, Steyn K, Bradshaw D, et al. Obesity in South Africa: the South African demographic and health survey. Obes Res 2002;10:1038-48.

39 Morrison C, McCook JG, Bailey BA. First trimester depression scores predict development of gestational diabetes mellitus in pregnant rural Appalachian women. J Psychosom Obstet Gynaecol 2016;37:21-5.

40 Byrn M, Penckofer S. The relationship between gestational diabetes and antenatal depression. J Obstet Gynecol Neonatal Nurs 2015:44:246-55.

$41 \mathrm{Knol}$ MJ, Twisk JWR, Beekman ATF, et al. Depression as a risk factor for the onset of type 2 diabetes mellitus. A meta-analysis. Diabetologia 2006;49:837-45.

42 Rotella F, Mannucci E. Depression as a risk factor for diabetes: a meta-analysis of longitudinal studies. J Clin Psychiatry 2013;74:31-7.

43 Gonzalez JS, Peyrot M, McCarl LA, et al. Depression and diabetes treatment nonadherence: a meta-analysis. Diabetes Care 2008;31:2398-403. 